\title{
Hypotensive Resuscitation
}

\author{
Jeremy B. Smith $\cdot$ Jean-Francois Pittet $\cdot$ \\ Albert Pierce
}

Published online: 9 May 2014

(C) Springer Science + Business Media New York 2014

\begin{abstract}
Hypotensive resuscitation is a component of damage control resuscitation, the evolving approach to resuscitation in severely injured trauma patients. Resuscitation strategies used in treating severely injured trauma patients have changed dramatically over the last 20 years. The purpose of this review is to examine the current literature pertaining to hypotensive resuscitation, explore its use in damage control resuscitation, and examine blood pressure management in the setting of severe trauma.
\end{abstract}

Keywords Trauma Hypotensive resuscitation . Hemostatic resuscitation - Damage control resuscitation

\section{Introduction}

Trauma is the leading cause of death in people 1-44 years old in the United States and the fifth leading cause of death for all ages [1]. The major cause of death in trauma patients is central nervous system injury. The second most common and possibly preventable factor in many of these deaths is exsanguination [2]. In recent years, damage control strategies have been increasingly used in the care of the severely injured trauma patient. As a part of a broader damage control approach, hypotensive resuscitation has

J. B. Smith $(\bowtie)$ J J.-F. Pittet · A. Pierce

Department of Anesthesiology, University of Alabama-

Birmingham, 619 19th Street South, JT 845, Birmingham,

AL 35249-6810, USA

e-mail: jbsmith@uab.edu

J.-F. Pittet

e-mail: pittetj@uab.edu

A. Pierce

e-mail: bpierce@uab.edu also become an increasingly utilized strategy in the management of many acutely injured patients [3•].

Over the past few decades, a dramatic shift has occurred with regards to the surgical treatment and resuscitation of severely injured trauma patients. Long definitive operations have slowly been replaced by multiple staged procedures in order to minimize surgical bleeding and allow for the patient's metabolic abnormalities to be addressed [4]. The approach to resuscitation has also changed in quantity, composition, and initial goals. The terms hypotensive resuscitation, damage control resuscitation, and hemostatic resuscitation have emerged to describe an approach to resuscitation that targets the trauma lethal triad of hypothermia, acidosis, and coagulopathy early in the care of the patient (Fig. 1).

\section{Systemic Effects of Hemorrhage and Trauma}

Trauma is both a local and systemic disease. When an injury occurs via direct damage to local tissues, solid organs, and blood vessels, multiple factors influence the amount of bleeding that follows [5]. The extent of the injury, pressure within and outside of the injured vessel, diameter of the vessel, vasoconstriction, and clot formation are all major local determinants of the degree of hemorrhage [6]. The systemic effects of trauma are the result of mediators released due to local tissue damage, ischemia from vessel thrombosis, and hypoperfusion [7, 8]. Many of these factors contribute to hyperfibrinolysis and anticoagulant effects. One such mechanism is hypoperfusioninduced systemic protein $\mathrm{C}$ activation. In this pathway, hypoperfusion causes an increase in thrombomodulin. Thrombomodulin then complexes with thrombin and this complex activates protein $\mathrm{C}$. The thrombomodulin- 
Fig. 1 Hypotensive resuscitation benefits within the lethal triad

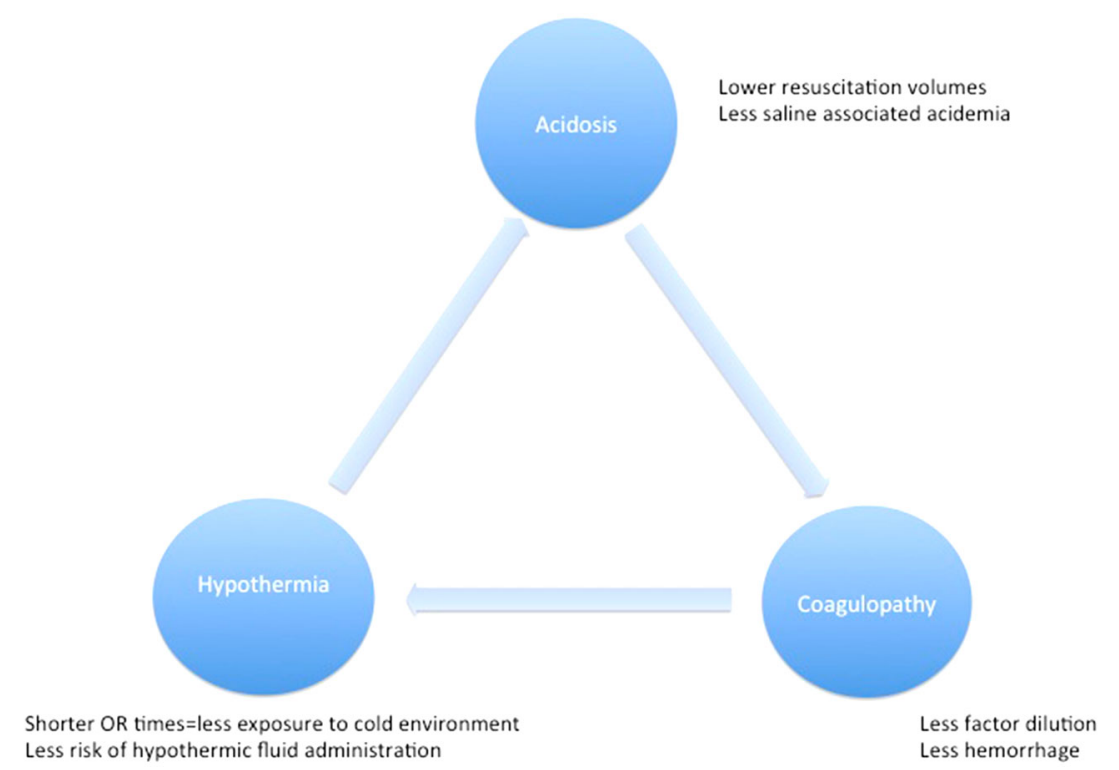

thrombin complex also decreases the amount of thrombin available for other thrombin functions, such as cleaving fibrinogen to fibrin and platelet activation. This contributes to the acute coagulopathy seen in one-fourth to one-third of severely traumatized patients [9]. Other mechanisms, such as endothelial glycocalyx disruption, fibrinogen depletion, intrinsic platelet dysfunction and Weibel-Palade body degranulation associated hyperfibrinolysis, are also contributory [10-14].

This trauma-induced coagulopathy (TIC) seems to be independent of the amount of blood loss on presentation. Even before significant blood loss has occurred, the coagulopathic response is already activated [9, 15]. As this coagulopathic process moves forward, there is consumption of coagulation factors and a cycle that perpetuates worsening coagulopathy and bleeding. As bleeding continues, tissues become more ischemic, releasing more detrimental factors, thus the process continues to spiral unless reversed by early intervention [5].

In addition to coagulation abnormalities, diverse systemic effects can occur due to tissue trauma, membrane disruption, and ischemia with resulting proinflammatory mediator release. Systemic inflammatory response syndrome (SIRS) is one such host response. SIRS involves a hyperinflammatory state that can cause organ dysfunction in organs not involved in the original injury. SIRS results from a complex interplay between inflammatory mediators and immune system response. Along with SIRS, endothelial cell dysfunction, leukocyte accumulation, and microcirculatory changes are all caused by inflammatory mediators and lead to worsening organ dysfunction and possibly multiple organ failure $[16,17]$.

\section{Resuscitation Strategies}

Traditional resuscitation strategies in the hypotensive trauma patient previously involved rapid administration of intravenous fluids, usually consisting of crystalloids, until a normalized blood pressure was reached. This was usually close to the patient's expected normal mean arterial pressure. But, over the past 20 years or more, the use of large volumes of crystalloids in the resuscitation of trauma patients has been questioned. In 1994, Bickell et. al. [37] performed a landmark prospective trial in which 598 patients with penetrating torso injuries were randomized into a delayed fluid group and a standard/immediate resuscitation group. This study found that survival was significantly improved in the delayed fluid group. In multiple animal studies, increasing volumes of intravenous (IV) fluids have been shown to increase bleeding during uncontrolled hemorrhage [18-21]. Large volumes of IV fluids increase mean arterial pressure by increasing cardiac preload and wall tension and increasing cardiac output. This increased cardiac output increases mean arterial pressure and also decreases local vasoconstriction, leading to more blood loss [5] [19]. A recent glue grant database analysis by Kasotakis et al. [22] reviewed over 1,700 blunt trauma patients and found that ventilator days and hospital length of stay correlated strongly with the amount of crystalloids given in the first $24 \mathrm{~h}$ after injury. A prospective study by Ley et al., found a correlation in patients receiving more than $1.5 \mathrm{~L}$ of crystalloid and increasing mortality [23]. Guidry et al. [24] found in that even in the setting of damage control resuscitation with high packed red blood cell (PRBC):fresh frozen plasma (FFP) 
transfusion ratios, higher amount of crystalloid IV fluids were associated with decreased survival.

In addition to changing the pressure dynamics of an injured vessel, initial large volume crystalloid resuscitation disrupts clot formation and stability [25]. In 1985, Hewson et al. [26] retrospectively studied 68 trauma patients who required massive transfusion. This study showed that increased partial thromboplastin time (PTT) values correlated with the amount of crystalloids given. This study also recommended that PRBC:FFP ratios approach 1:1. Another, more recent, retrospective study on patients undergoing major abdominal procedures done by Barak et al. [27] found that patients who were given more than $3 \mathrm{~L}$ of crystalloids had worsened coagulation function. Trauma-induced coagulopathy is currently a very active area of research. There is a complex interaction between biochemical mediators, pro and anticoagulant factors, and inflammation that is incompletely understood. This interaction also seems to be influenced by the type and amount of resuscitation fluids, with a trend towards better survival with decreasing amounts of crystalloids and lower blood pressures in certain trauma populations [22, 24, 28, 29].

Many other organ systems seem to be affected by large volume IV crystalloid use. In a randomized blinded study, Brandstrup et al. studied 172 patients undergoing major colorectal surgery. This study found a significant reduction in cardiopulmonary complications in the fluid restricted group. Also of note, there were four deaths in the standard fluid group versus none in the restricted group [30]. In trauma patients, the use of large volume has been associated with an increased incidence of acute respiratory distress syndrome, bacteremia, surgical site infections, and abdominal compartment syndrome [22].

Regarding prehospital IV crystalloid administration, a recent analysis of the Prospective, Observational, Multicenter, Massive Transfusion Trial (PROMMTT) data found that pre-hospital IV crystalloid use increased survival over those who received no pre-hospital fluids. In this study, patients received an average of $700 \mathrm{cc}$ of IV crystalloid in the pre-hospital setting. Of note, these patients had decreased hematocrit/hemoglobin levels and decreased fibrinogen and platelet levels. They were also noted to have increased prothrombin times/partial thromboplastin times and international normalized ratio (INR) levels when compared to patients who received no fluid [31]. Another recent study done by Brown et al. found that hypotensive blunt trauma patients who received more than $500 \mathrm{cc}$ prehospital IV crystalloids had improved survival. But in those that were not hypotensive, over $500 \mathrm{cc}$ of fluid decreased survival [32•]. Another large retrospective study from 2011 using data from the National Trauma Databank showed detrimental effects of pre-hospital fluids in trauma patients. However, no data or stratification based on IV fluid volumes were available [33]. In regards to these conflicting findings, there is a very complex interplay between the type of trauma, nature of the injury, length of transport, changes in mean arterial pressure, and type of resuscitation. The Brown et al. [32•] study above seems to suggest that a goal-directed approach to pre-hospital fluid might be beneficial. The question of how much and in what clinical scenarios is crystalloid IV fluid beneficial versus harmful is currently a topic for debate and has not been definitively answered.

\section{Hypotensive Resuscitation as Part of Damage Control Management}

Hypotensive resuscitation consists of a restrictive approach to the administration of IV fluids and blood, with the goal of keeping mean arterial pressures (MAP) high enough for essential organ perfusion but lower than normal in order to limit bleeding. Multiple animal studies have shown this approach to be beneficial in acute hemorrhage. These studies show less blood loss at lower blood pressures [18, $19,34,35]$. In the actively bleeding patient, these lower MAPs translate into less acute blood loss and less coagulopathy [28]. Because of this, hypotensive resuscitation is rapidly replacing the high volume resuscitation standard of care in the acute phase of trauma care (Fig. 2).

Over the past 20 years, surgical goals have shifted from definitive operations to a more "damage control" approach. In 1993, Rotondo and Schwab reported an increase in survival from 11 to $77 \%$ using multiple staged laparotomies to treat penetrating truncal trauma [36]. Since then, the damage control approach has gradually become the treatment of choice in many types of trauma and is gaining acceptance in other areas such as vascular surgery and abdominal sepsis. Using this approach, trauma surgeons opt for multiple staged laparotomies in which bleeding and contamination are halted, but definitive repair is not undertaken until resuscitative and coagulopathic concerns are addressed [4].

Along with the shift in initial surgical goals, there has been a gradual shift from high volume resuscitations to a more restrictive approach, especially in the initial phase of treatment. Instead of the large volume, supraphysiologic goals of previous years, a different resuscitation strategy has emerged. The term "damage control resuscitation" (DCR) describes an approach to resuscitation that aims to avoid and treat the acidosis, hypothermia, and coagulopathy triad. Military clinicians first used the term DCR around 2005 [38]. In 2007, Holcomb outlined DCR as an approach to treating severely injured soldiers in Iraq. In this editorial, he noted the two main parts of DCR. The first was permissive hypotension with a goal of systolic blood 
Fig. 2 Effects of hypotensive resuscitation on coagulation and blood loss

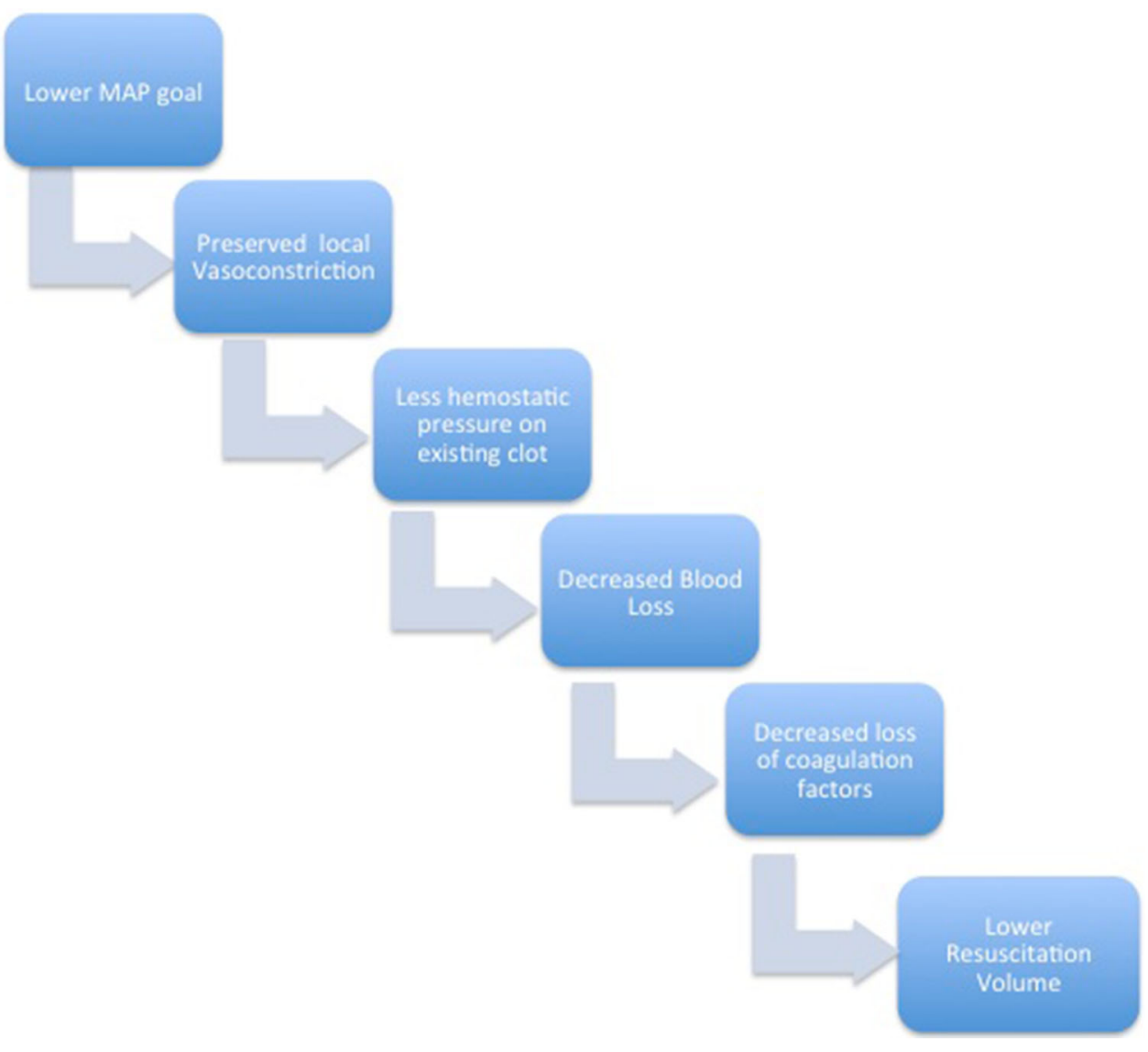

pressure (BP) of about $90 \mathrm{mmHg}$. The second was the use of FFP early in resuscitation, in a 1:1 ratio with $\mathrm{PRBC}$, and as the main resuscitative fluid [39]. In addition to these two goals, limiting crystalloid IV fluids has also become a major part of DCR [5].

A few recent studies have looked at combining damage control surgical approaches with damage control resuscitation. In 2010, Duchesne et al. looked at massively transfused ( $>10 \mathrm{U}$ PRBC in the first $24 \mathrm{~h}$ ) damage control laparotomy patients in a 4-year period during which DCR approaches were implemented. This study found a survival advantage in those patients treated after DCR became standard [40]. In a retrospective cohort study, Cotton et al. compared two groups of patients who had undergone a damage control laparotomy and surgical management. One group received a damage control approach to resuscitation and one did not. The DCR group had less evidence of the lethal triad post operatively, received less blood products, and had a significantly increased survival at $24 \mathrm{~h}$ and at 30 days [38]. Another retrospective study by Duke et al. had similar findings. This study compared a standard and restrictive fluid group. Patients in the restrictive fluid group received less than $150 \mathrm{cc}$ of crystalloid. The patients in this restrictive group had a lower intraoperative mortality, a shorter length of hospital stay and a lower overall mortality [3•]. The findings of these retrospective studies suggest that damage control resuscitation is an effective approach when treating patients that are high risk for massive transfusion. In practice, DCR seems to be gaining acceptance as a better alternative to classical crystalloid-based resuscitations.

\section{Blood Pressure Management During Hemorrhage}

There are typically two phases involved in trauma resuscitation. The early phase consists of resuscitation while bleeding is ongoing. The late phase is the period after bleeding has been controlled [41]. As mentioned above, elevation of MAP during acute hemorrhage increases blood loss. Multiple animal studies have also shown a survival benefit with both permissive hypotension and resuscitation goals below normal mean arterial pressures. These studies consistently show less blood loss at lower MAPs, increased survival time in uncontrolled hemorrhage, and increased blood requirements in the groups of animals resuscitated to normal or near normal MAPs or cardiac indexes [42-44]. A study by Li et al. found better survival and less blood loss in rats at mean arterial pressures between 50 and 
$60 \mathrm{mmHg}$. MAPs below $50-60 \mathrm{mmHg}$ were associated with more end organ dysfunction and lower survival rates. MAPs above 50-60 led to more blood loss and again more end organ dysfunction [34].

Unfortunately, in humans, there is very little definitive data guiding optimal mean arterial pressure management during permissive hypotension. In the non-trauma population, controlled hypotension has been used for procedures as a way to decrease blood loss and allow for better arthroscopic visualization by the surgeon. Although there is some evidence that blood loss is decreased and that in certain situations controlled hypotension may be beneficial, systolic pressures of $80-90 \mathrm{mmHg}$ or MAPs of around $50 \mathrm{mmHg}$ are generally recommended in patients that do not have contraindications to hypotensive management [45, 46].

In trauma patients, optimal blood pressure management during active hemorrhage is still unclear. In 2002, Dutton et al. evaluated 110 patients who were randomized into a conventional group with a target systolic pressure of $110 \mathrm{~mm} \mathrm{Hg}$ and another group with a target of $70 \mathrm{mmHg}$. This study found no difference in survival between the groups [47]. A recent 2013 study by Morrison et al. found a significant decrease in mortality in the immediate postoperative period and a trend towards a decrease in mortality at 30 days in the hypotensive arm. This study randomized patients into two arms based on mean arterial pressures. One arm used standard resuscitation in order to obtain a MAP of $65 \mathrm{mmHg}$. The other arm used a hypotensive resuscitation strategy with a target mean of $50 \mathrm{mmHg}$. Although the actual difference in mean arterial pressures in the two groups was not statistically significant (64 vs. $68 \mathrm{mmHg}$ ), the lower mean group received less blood products, were less coagulopathic, and, in penetrating injuries, had less blood loss [28]. These two studies and the Bickell study mentioned above are the only prospective studies that have looked at hypotensive resuscitation or permissive hypotension in trauma patients. Both the Bickell and the Morrison studies found increased survival with permissive hypotension. Currently, most major civilian trauma centers have adopted some form of hypotensive resuscitative techniques into practice.

In patients with a suspected head injury, hypotensive resuscitation is controversial. The actively bleeding patient with a concurrent head injury presents a clinical dilemma. Current guidelines recommend a systolic blood pressure of at least $90 \mathrm{mmHg}$ in order to maintain cerebral perfusion pressure, and multiple studies have found hypotension to be an independent predictor of mortality in head injured patients [48]. In one study, hypotension was associated with a $150 \%$ increase in mortality in the head injured patient [49]. Unfortunately, this situation presents two clinical scenarios in the same patient: active bleeding that would seem to benefit from a hypotensive approach and a head injury that is very sensitive to mean arterial pressure in relation to cerebral perfusion. Because of this sensitivity, if possible, systolic BP of $90 \mathrm{mmHg}$ should be maintained in patients suspected of a head injury [50].

\section{Conclusion}

Severe traumatic injury is a major cause of death and disability. A dramatic change has occurred over the past 20 years in the treatment of severely injured patients. Many patients seem to survive injuries today that would have been fatal 10-15 years ago. Unfortunately, prospective trials in trauma patients are logistically very difficult. Retrospective and animal studies strongly support the use of damage control and hypotensive resuscitation in severely injured patients. Of the three prospective studies dealing with permissive hypotension, two showed increased survival, but none showed harm. Currently, this is a very active area of research with many unanswered questions. Questions regarding component ratios, the role of crystalloids in hemostatic resuscitation, trauma-induced coagulopathy and treatment, and optimal mean arterial pressures during hemorrhage, are all complex and have not been completely answered. The recently finished multicenter PROPPR trial (Pragmatic Optimized Platelet and Plasma Ratios) will hopefully give some insights into the optimal use of blood components in resuscitation.

\section{Compliance with Ethics Guidelines}

Conflict of Interest Jeremy B. Smith, Jean-Francois Pittet, and Albert Pierce declare that they have no conflict of interest.

Human and Animal Rights and Informed Consent This article does not contain any studies with human or animal subjects performed by any of the authors.

\section{References}

Papers of particular interest, published recently, have been highlighted as:

- Of importance

1. Minino AM, Murphy SL. Death in the United States, 2010. NCHS Data Brief. 2012;99:1-8.

2. Demetriades D, et al. Trauma fatalities: time and location of hospital deaths. J Am Coll Surg. 2004;198(1):20-6.

3. Duke MD, et al. Restrictive fluid resuscitation in combination with damage control resuscitation: time for adaptation. J Trauma Acute Care Surg. 2012;73(3):674-8. This study is a retrospective analysis that compares a standard resuscitation group and a 
restrictive resuscitation group after damage control surgical techniques became standard.

4. Waibel BH, Rotondo MM. Damage control surgery: it's evolution over the last 20 years. Rev Col Bras Cir. 2012;39(4):314-21.

5. Dutton RP. Haemostatic resuscitation. $\mathrm{Br} \mathrm{J}$ Anaesth. 2012;109(Suppl 1):i39-46.

6. Shaftan GW, et al. Fundamentals of physiologic control of arterial hemorrhage. Surgery. 1965;58(5):851-6.

7. Dutton RP. Current concepts in hemorrhagic shock. Anesthesiol Clin. 2007;25(1):23-34.

8. Chaudry IH, Bland KI. Cellular mechanisms of injury after major trauma. Br J Surg. 2009;96(10):1097-8.

9. Brohi $\mathrm{K}$, et al. Acute coagulopathy of trauma: hypoperfusion induces systemic anticoagulation and hyperfibrinolysis. J Trauma. 2008;64(5):1211-7.

10. Chambers LA, Chow SJ, Shaffer LE. Frequency and characteristics of coagulopathy in trauma patients treated with a low- or high-plasma-content massive transfusion protocol. Am J Clin Pathol. 2011;136(3):364-70.

11. Hiippala ST, Myllyla GJ, Vahtera EM. Hemostatic factors and replacement of major blood loss with plasma-poor red cell concentrates. Anesth Analg. 1995;81(2):360-5.

12. Ostrowski SR, Johansson PI. Endothelial glycocalyx degradation induces endogenous heparinization in patients with severe injury and early traumatic coagulopathy. J Trauma Acute Care Surg. 2012;73(1):60-6.

13. Wohlauer MV, et al. Early platelet dysfunction: an unrecognized role in the acute coagulopathy of trauma. J Am Coll Surg. 2012;214(5):739-46.

14. Whitcomb DC, et al. Angiopoietin-2, a regulator of vascular permeability in inflammation, is associated with persistent organ failure in patients with acute pancreatitis from the United States and Germany. Am J Gastroenterol. 2010;105(10):2287-92.

15. Hess JR, et al. The prevalence of abnormal results of conventional coagulation tests on admission to a trauma center. Transfusion. 2009;49(1):34-9.

16. Keel M, Trentz O. Pathophysiology of polytrauma. Injury. 2005;36(6):691-709.

17. Lenz A, Franklin GA, Cheadle WG. Systemic inflammation after trauma. Injury. 2007;38(12):1336-45.

18. Lin GS, et al. Target blood pressure for hypotensive resuscitation. Injury. 2013;44(12):1811-5.

19. Burris D, et al. Controlled resuscitation for uncontrolled hemorrhagic shock. J Trauma. 1999;46(2):216-23.

20. Bickell WH, et al. The detrimental effects of intravenous crystalloid after aortotomy in swine. Surgery. 1991;110(3):529-36.

21. Riddez L, Johnson L, Hahn RG. Central and regional hemodynamics during crystalloid fluid therapy after uncontrolled intraabdominal bleeding. J Trauma. 1998;44(3):433-9.

22. Kasotakis G, et al. Aggressive early crystalloid resuscitation adversely affects outcomes in adult blunt trauma patients: an analysis of the Glue Grant database. J Trauma Acute Care Surg. 2013;74(5):1215-21 (discussion 1221-2).

23. Ley EJ, et al. Emergency department crystalloid resuscitation of $1.5 \mathrm{~L}$ or more is associated with increased mortality in elderly and nonelderly trauma patients. J Trauma. 2011;70(2):398-400.

24. Guidry $\mathrm{C}$, et al. Initial assessment on the impact of crystalloids versus colloids during damage control resuscitation. J Surg Res. 2013;185(1):294-9.

25. Torres LN, et al. Evaluation of resuscitation fluids on endothelial glycocalyx, venular blood flow, and coagulation function after hemorrhagic shock in rats. J Trauma Acute Care Surg. 2013;75(5):759-66.

26. Hewson JR, et al. Coagulopathy related to dilution and hypotension during massive transfusion. Crit Care Med. 1985;13(5):387-91.
27. Barak M, et al. Fluid administration during abdominal surgery influences on coagulation in the postoperative period. Curr Surg. 2004;61(5):459-62.

28. Morrison CA, et al. Hypotensive resuscitation strategy reduces transfusion requirements and severe postoperative coagulopathy in trauma patients with hemorrhagic shock: preliminary results of a randomized controlled trial. J Trauma. 2011;70(3):652-63.

29. Wang $\mathrm{CH}$, et al. Liberal Versus Restricted Fluid Resuscitation Strategies in Trauma Patients: a systematic review and metaanalysis of randomized controlled trials and observational studies. Crit Care Med. 2013.

30. Brandstrup B, et al. Effects of intravenous fluid restriction on postoperative complications: comparison of two perioperative fluid regimens: a randomized assessor-blinded multicenter trial. Ann Surg. 2003;238(5):641-8.

31. Hampton DA, et al. Prehospital intravenous fluid is associated with increased survival in trauma patients. J Trauma Acute Care Surg. 2013;75(1 Suppl 1):S9-15.

32. • Brown, J.B., et al., Goal-directed resuscitation in the prehospital setting: a propensity-adjusted analysis. J Trauma Acute Care Surg. 2013;74(5):1207-12; discussion 1212-4. This study illustrates the possible utility of a goal directed approach to initial trauma resuscitation.

33. Spoerke N, et al. Crystalloid resuscitation improves survival in trauma patients receiving low ratios of fresh frozen plasma to packed red blood cells. J Trauma. 2011;71(2 Suppl 3):S380-3.

34. Li $\mathrm{T}$, et al. Ideal permissive hypotension to resuscitate uncontrolled hemorrhagic shock and the tolerance time in rats. Anesthesiology. 2011;114(1):111-9.

35. Zhang YM, et al. Effect of hypotensive resuscitation with a novel combination of fluids in a rabbit model of uncontrolled hemorrhagic shock. PLoS ONE. 2013;8(6):e66916.

36. Rotondo MF, et al. 'Damage control': an approach for improved survival in exsanguinating penetrating abdominal injury. J Trauma. 1993;35(3):375-82.

37. Bickell WH, et al. Immediate versus delayed fluid resuscitation for hypotensive patients with penetrating torso injuries. $\mathrm{N}$ Engl J Med. 1994;331(17):1105-9.

38. Cotton BA, et al. Damage control resuscitation is associated with a reduction in resuscitation volumes and improvement in survival in 390 damage control laparotomy patients. Ann Surg. 2011;254(4):598-605.

39. Holcomb JB. Damage control resuscitation. J Trauma. 2007;62(6 Suppl):S36-7.

40. Duchesne JC, et al. Damage control resuscitation in combination with damage control laparotomy: a survival advantage. J Trauma. 2010;69(1):46-52.

41. Duchesne JC, et al. Hemostatic resuscitation during surgery improves survival in patients with traumatic-induced coagulopathy. J Trauma. 2009;67(1):33-7 (discussion 37-9).

42. Owens TM, et al. Limiting initial resuscitation of uncontrolled hemorrhage reduces internal bleeding and subsequent volume requirements. J Trauma. 1995;39(2):200-7 (discussion 208-9).

43. Durusu M, et al. Comparison of permissive hypotensive resuscitation, low-volume fluid resuscitation, and aggressive fluid resuscitation therapy approaches in an experimental uncontrolled hemorrhagic shock model. Ulus Travma Acil Cerrahi Derg. 2010;16(3):191-7.

44. Rezende-Neto JB, et al. Rabbit model of uncontrolled hemorrhagic shock and hypotensive resuscitation. Braz J Med Biol Res. 2010;43(12):1153-9.

45. Paul JE, et al. Deliberate hypotension in orthopedic surgery reduces blood loss and transfusion requirements: a meta-analysis of randomized controlled trials. Can J Anaesth. 2007;54(10):799-810. 
46. Dutton RP. Controlled hypotension for spinal surgery. Eur Spine J. 2004;13(Suppl 1):S66-71.

47. Dutton RP, Mackenzie CF, Scalea TM. Hypotensive resuscitation during active hemorrhage: impact on in-hospital mortality. J Trauma. 2002;52(6):1141-6.

48. Bratton SL, et al. Guidelines for the management of severe traumatic brain injury. I. Blood pressure and oxygenation. J Neurotrauma. 2007;24(Suppl 1):S7-13.
49. Chesnut RM, et al. The role of secondary brain injury in determining outcome from severe head injury. J Trauma. 1993;34(2): 216-22.

50. Tobin JM, et al. Hypotensive resuscitation in a head-injured multi-trauma patient. J Crit Care. 2013;29(2):313 e1-e5. 